\title{
Pharmacological Activities of Sijunzi Decoction Which Are Related to Its Antioxidant Properties
}

\author{
Lei Ye, ${ }^{1}$ Jianwei Gong, ${ }^{1}$ Yonghua Wang, ${ }^{2}$ and Xiling Sun ${ }^{1}$ \\ ${ }^{1}$ Department of Chinese Medicine Integrated with Western Medicine, Binzhou Medical University, Yantai, Shandong 264003, China \\ ${ }^{2}$ School of Biosciences, Northwest A\&F University, Xi'an, Shanxi 710000, China \\ Correspondence should be addressed to Xiling Sun; sunxilingsun@sohu.com
}

Received 18 May 2014; Accepted 5 July 2014; Published 23 July 2014

Academic Editor: Qiusheng Zheng

Copyright (c) 2014 Lei Ye et al. This is an open access article distributed under the Creative Commons Attribution License, which permits unrestricted use, distribution, and reproduction in any medium, provided the original work is properly cited.

\begin{abstract}
This paper introduces the antioxidant constituents and pharmacological effects of Sijunzi decoction by looking up literatures in recent years. Sijunzi decoction is composed of Ginseng, Atractylodes, Tuckahoe, and Glycyrrhiza. The antioxidant ingredients of Sijunzi decoction include paeonol, dauricine, naringin, and isoliquiritigenin. The study has proved that it possesses wide pharmacological effects of anticardiovascular diseases, antinervous system disease, antidiabetes, antimetabolic syndrome, and antitumor. Research on the antioxidant components of Sijunzi decoction and their targets is a promising study area in the future.
\end{abstract}

\section{Introduction}

Phagocytic cells release a significant amount of reactive oxygen species (ROS) through respiratory burst under the stimulus of pathogenic microorganisms when "spleen qi deficiency" occurs, which is often associated with gastrointestinal inflammation. Through NADPH oxidation, ROS can cause oxidative damage and further aggravate the inflammation. Studies have shown that Sijunzi decoction has evident antioxidant effects [1] and can eliminate or reduce biological membrane lipid peroxidation caused by free radicals [2]. Moreover, Sijunzi decoction can increase superoxide dismutase (SOD) activity and reduce MDA generation to speed up the elimination of free radicals and other harmful substances to protect biological macromolecules from damage and improve the DNA repair. Sijunzi decoction has an antioxidant effect because it contains a wide variety of free radical scavenging molecules. These antioxidant compounds possess anti-inflammatory, antiatherosclerotic, antitumor, antimutagenic, anticarcinogenic, or antibacterial properties. The antioxidants derived from Sijunzi decoction are summarized in this study. The source of antioxidants discussed in this study is shown in Table 1.

\section{The Role of Antioxidants from Sijunzi Decoction}

2.1. Antioxidants of Anticardiovascular Disease. Several components of Sijunzi soup have antioxidant activity and decrease the occurrence of cardiovascular diseases. Studies have shown that isoliquiritigenin, which is a natural antioxidant derived from liquorice, has a significant role in anticardiovascular disease through activation of the AMP-activated protein kinase (AMPK) and ERK signaling pathways and balance of cellular redox status [3]. Naringin derived from liquorice, which is the second most important antioxidant component, inhibits reactive oxygen species- (ROS-) activated MAPK pathway involved in high glucose-induced injuries of $\mathrm{H} 9 \mathrm{c} 2$ cardiac cells [4]. Naringin mitigates hypertension and thrombosis by increasing the bioavailability of nitrogen oxide (NO) and protecting the endothelial function from ROS [5]. Moreover, naringin can improve redox-sensitive myocardial ischemia reperfusion injury [6]. Glabridin, which is derived from liquorice and is responsible for its antioxidative characteristics in low-density lipoprotein (LDL) oxidation, is also important [7]. Isorhamnetin, which is also derived from liquorice, can inhibit the $\mathrm{H}_{2} \mathrm{O}_{2}$-induced activation of the 
TABLE 1

\begin{tabular}{|c|c|}
\hline $\begin{array}{l}\text { Source of } \\
\text { ingredient }\end{array}$ & Antioxidant ingredient \\
\hline Ginseng & Paeonol, dauricine, pancratistatin \\
\hline Licorice & $\begin{array}{l}\text { Isoliquiritigenin, naringin, glabridin, isorhamnetin, } \\
\text { calycosin, pinocembrin, nicotiflorin, liquiritigenin, } \\
\text { kaempferol, licoflavone, isobavachalcone, morusin, } \\
\text { licochalcone A, licochalcone B, rutin, quercetin }\end{array}$ \\
\hline
\end{tabular}

intrinsic apoptotic pathway through ROS scavenging and ERK inactivation. Therefore, isorhamnetin is a promising reagent for the treatment of ROS-induced cardiomyopathy [8].

Paeonol, which is mainly collected from ginseng, affects the development of cardiovascular tissues. Paeonol has antioxidant and anti-inflammatory properties, which can be developed for use in anti-inflammatory and vascular disorders [9]. The therapeutic mechanism of paeonol prevents monocyte adhesion onto vascular endothelial cells (VECs) induced by ox-LDL [10]. Paeonol has a protective effect on the hypoxia/reoxygenation damage of myocardial cells; therefore, it can prevent myocardial infarction induced by isoproterenol in rats [11]. Paeonol can also decrease oxidative injury and repair blood vessel endothelium, as well as prevent the development of coronary diseases [12].

2.2. Antioxidants against Diabetes and Metabolic Syndrome. Metabolic syndrome is a low-grade inflammatory state, where oxidative stress is involved. Some of the antioxidants extracted from Sijunzi decoction exhibit antidiabetes activities or metabolic syndrome reversal. Isoliquiritigenin can significantly decrease the level of blood glucose [13]. Naringin exhibits significant antidiabetic effects by potentiating the antioxidant defense system and by suppressing proinflammatory cytokine production in a rat model of T2DM [14]. Naringin reverses the metabolic syndrome by decreasing inflammatory cell infiltration and plasma lipids; the process mitigates oxidative stress and improves mitochondrial function [15]. Glabridin, which is a major active flavonoid in Glycyrrhiza glabra (licorice), improves learning and memory in mice. Glabridin reverses learning and memory defects in diabetic rats. Glabridin treatment partially improves the reduced body weight and hyperglycemia of diabetic rats. Their mechanisms may be related to the combination of antioxidant, neuroprotective, and anticholinesterase properties of glabridin [16] or as an activator of AMP-activated protein kinase (AMPK) [17].

2.3. Antioxidants of Antineurological Diseases. Clinically, Sijunzi decoction is often used to treat several neurological diseases. Calycosin, which is derived from licorice, has a neuroprotective effect against cerebral ischemia/reperfusion injury through its antioxidant effects [55]. Isoliquiritigenin protects HT22 hippocampal neuronal cells from oxidative stress-induced glutamate. The mechanisms are related to the reversed ROS production and mitochondrial depolarization induced by glutamate, as well as the regulation expression of the apoptotic regulators, $\mathrm{Bcl}-2$ and $\mathrm{Bax}$ [31]. Isoliquiritigenin can protect dopaminergic cells from oxidative injury and prevent $\mathrm{A} \beta(25-35)$-induced neuronal apoptotic death by interfering with the increase of $\left[\mathrm{Ca}^{+}\right]$and ROS [32]. Liquiritigenin is derived from licorice; this compound improves behavioral performance and attenuates neuronal loss in the brain of rats [64]. Pinocembrin derived from licorice has a significant role in neurovascular protection by protecting the cerebral ischemia and increasing the viability of the mitochondrial membrane $[57,58,80,81]$. Pinocembrin abrogates the effects of the neurotoxin 1-methyl-4phenylpyridinium, which mimics Parkinson's disease with an elevation of intracellular ROS level and apoptotic death [82]. This phenomenon might be another mechanism for pinocembrin in mitigating nervous system diseases.

Nicotiflorin, which is derived from licorice, markedly reduces brain infarct volume and neurological deficits immediately following its administration after the onset of ischemia. Nicotiflorin also protects against memory dysfunction, energy metabolism failure, oxidative stress in multiinfarct dementia model rats, cerebral ischemic damage, aluminum chloride-induced cognitive dysfunction, and mitochondrial oxidative damage $[61,62]$. Investigations about the protective mechanism of kaempferol glycosides showed the involvement of their antioxidative activity, blockage of caspase cascades, attenuation of NMDA-induced neuronal toxicity, inhibition of monoamine oxidase (MAO), excessive NO production, and others [63].

Naringin may be beneficial in mitigating 3-NP-induced neurodegeneration through its antioxidant and antiapoptotic effects $[39,40]$.

Glabridin has a neuroprotective effect because of the modulation of multiple pathways associated with apoptosis. Glabridin significantly attenuates the level of brain malonyldialdehyde (MDA) in MCAO rats; however, it elevates the levels of two endogenous antioxidants in the brain, namely, SOD and reduced glutathione [48].

Dauricine is derived from ginseng and has cerebral ischemia-reperfusion injury protection effect, which may be related to its inhibition of neuron apoptosis [22]. Paeonol mitigates neuronal damage not only by decreasing ROS overgeneration, but also by regulating expression of apoptosis proteins and neurotrophic factors $[18,19]$.

2.4. Antioxidants of Antirespiratory Diseases. Three components from Sijunzi soup have significant roles in preventing respiratory diseases.

Naringin has antitussive, anti-AHR, and anti-inflammation effects on chronic cigarette smoke exposure-induced chronic bronchitis in guinea pigs; this compound improves SOD activity in lung tissue and increases the content of lipoxin A4 in bronchoalveolar lavage fluid (BALF) in a guinea pig model for chronic bronchitis [41, 42]. Dauricine induces glutathione depletion and apoptosis in lungs of CD-1 mice and in cultured human lung cells [23]. Liquiritigenin can protect human lung cells (A549) from a hemolysin-mediated injury [65]. 
2.5. Antioxidants against Digestive System Diseases. Several components of Sijunzi soup are also involved in antidigestive system diseases.

Liquiritigenin has a choleric effect and exhibits the ability to induce hepatic transporters and phase-II enzymes, which are a group of antioxidant systems that function against oxidative stress, carcinogenesis, mutagenesis, and other forms of toxicity [66]. Isoliquiritigenin, which is similar with liquiritigenin, can repress LXR $\alpha$-dependent hepatic steatosis through JNK1 inhibition and protect hepatocytes from oxidative injury inflicted by fat accumulation [33].

The induction of $\mathrm{HO}$ activity by pinocembrin has a protective effect against hepatic damage associated with oxidative stress in rats. Pinocembrin challenges hepatocarcinogens and may exhibit anticarcinogenic effects [59]. Dauricine is subject to oxidative bioactivation in human liver microsomes in vitro and in rats in vivo [24].

Licoflavone, isobavachalcone, and quercetin are derived from licorice. Licoflavone feeding suppresses gastric mucosa injury and protects and restores injured mucosa in rats with chronic superficial gastritis. These effects are related with the upregulation of serum PGE2 levels [69]. Isobavachalcone shows broad antioxidative activities in rat liver microsomes and mitochondria [70]. Quercetin has beneficial effects on liver fibrosis in rats by enhancing antioxidant enzyme activity and reducing the prooxidant effects [79].

Several studies have demonstrated that isorhamnetin is efficient in protecting hepatocytes against oxidative stress by Nrf2 activation and in inducing the expression of its downstream genes [50].

Paeonol can inhibit HSC proliferation and induce mitochondrial apoptosis by disrupting the NF- $\kappa \mathrm{B}$ pathway, which is probably the mechanism of paeonol reduction of liver fibrosis [20].

2.6. Antioxidants of Antitumors. Natural compounds isolated from Sijunzi decoction, which are rich sources of novel anticancer drugs, have gained increasing interest.

Isoliquiritigenin significantly inhibits the proliferation of C4-2 prostate cancer cells in IEC-6 normal epithelial cells in vitro, increases intracellular ROS levels, and causes SKOV-3 and hela cell apoptosis [34-36]. The prodifferentiation effect of isoliquiritigenin on HL-60, particularly the role of redox homeostasis in regulating HL-60 cell differentiation by modulation of the Nrf2/ARE pathway, has been investigated $[37,38]$.

Licochalcone A and licochalcone $\mathrm{B}$ are derived from licorice. Licochalcone A has potent antitumor effects in prostate, breast, and bladder cancer, as well as in leukemia cell lines [74]. A study has shown that the induction of endoplasmic reticulum stress through a PLC $\gamma 1-, \mathrm{Ca}^{2+}-$, and ROS-dependent pathways may be significant when licochalcone A induces apoptosis in HepG2 hepatocellular carcinoma cells [75]. Furthermore, licochalcone B inhibits the concentration-dependent bladder cancer cell proliferation; this antiproliferative effect is caused by the induction of Sphase arrest and apoptotic cell death [77].
Dauricine can inhibit the proliferation activity of urinary tract tumor cells. By inhibiting the NF- $\kappa$ B signaling pathway in colon cancer cells, dauricine inhibits proliferation and invasion of the cancer $[25,26]$. Furthermore, dauricine inhibits human breast cancer angiogenesis by suppressing hypoxia inducible factor- $1 \alpha$ (HIF- $1 \alpha$ ) protein accumulation and vascular endothelial growth factor (VEGF) expression [27].

Naringin can induce receptor death and mitochondriamediated apoptosis in human cervical cancer cells [43]. Naringin reduces the growth potential of human triplenegative breast cancer cells by targeting the $\beta$-catenin signaling pathway [44]. Naringin is effective in reducing the number of preneoplastic lesions in rats exposed to 1,2dimethylhydrazine. Several of these effects may be caused by reduced cellular proliferation and tissue levels of iron and the recovery of antioxidant mineral levels induced by this flavonoid [45].

Pancratistatin is derived from ginseng and may be a novel mitochondria-targeting compound that selectively induces apoptosis in cancer cells and significantly reduces tumor growth [28, 29]. Pancratistatin treatment in cancer cell lines results in increased ROS production and reduction of mitochondrial membrane potential [30]. Morusin, which is derived from licorice, significantly inhibits the growth and clonogenicity of HT-29 human colorectal cancer cells, as well as the human cervical cancer stem cell growth and migration $[72,73]$.

Calycosin is an indispensable element in antitumor activities. Calycosin inhibits breast cancer growth and is obtained by estrogen receptor (ER) $\beta$-mediated regulation of the IGF-1R signaling pathways and miR-375 expression [56]. Isorhamnetin exhibits antioxidant and antiproliferative activities in a variety of cancer cell lines [51-53].

Glabridin is a novel anticancer agent for treating breast cancer in three different manners, namely, inhibition of migration, invasion, and angiogenesis [49]. Pinocembrin triggers Bax-dependent mitochondrial apoptosis in colon cancer cells [60]. Isobavachalcone induces apoptotic cell death in neuroblastoma through the mitochondrial pathway and is not cytotoxic against normal cells [71]. Paeonol has significant growth-inhibitory and apoptosis-inducing effects in gastric cancer cells, both in vitro and in vivo [21].

2.7. Antioxidants against Inflammation. The components of Sijunzi soup have a significant role against inflammation.

Naringin is an effective anti-inflammatory compound for attenuating the chronic pulmonary neutrophilic inflammation in CS-induced rats. The oxidative stress caused by cigarette smoke (CS) exposure increases inflammatory cell influxes in the lungs, followed by lipid peroxidation, and increases proinflammatory cytokines, such as the tumor necrosis factor- $\alpha$ [46]. Liquiritigenin has an antiinflammatory effect, as shown by the inhibition of nitric oxide and tumor necrosis factor-production, which is induced by the lipopolysaccharides in macrophages [67]. Licochalcone $\mathrm{A}$ protects $\mathrm{BALB} / \mathrm{c}$ mice from lipopolysaccharide(LPS-) induced endotoxin shock by inhibiting the production 
TABle 2

\begin{tabular}{|c|c|c|c|}
\hline $\begin{array}{l}\text { Antioxidant } \\
\text { ingredient }\end{array}$ & Structure of compound & Pharmacological activity & References \\
\hline Paeonol & & $\begin{array}{l}\text { (1) Antioxidant and anti-inflammatory } \\
\text { properties } \\
\text { (2) Treatment for vascular disorders } \\
\text { (3) Protection against atherosclerosis. } \\
\text { (4) Neuroprotection } \\
\text { (5) Protection against hypoxia/reoxygenation } \\
\text { damage } \\
\text { (6) Growth-inhibitory and apoptosis-inducing } \\
\text { effects on cancer cells }\end{array}$ & {$[9-12,18-21]$} \\
\hline Dauricine & & $\begin{array}{l}\text { (1) Inhibition of neuron apoptosis } \\
\text { (2) Induction of glutathione depletion and } \\
\text { apoptosis in cancer cells } \\
\text { (3) Inhibition of tumor cell proliferation } \\
\text { (4) Inhibition of cancer angiogenesis } \\
\text { (5) Induction of tumor cell apoptosis }\end{array}$ & {$[22-27]$} \\
\hline Pancratistatin & $\mathrm{OH}$ & $\begin{array}{l}\text { (1) Increased reactive oxygen species (ROS) } \\
\text { production } \\
\text { (2) Induction of mitochondrial membrane } \\
\text { collapse } \\
\text { (3) Potential induction of tumor apoptosis } \\
\text { (4) Reduction of tumor growth }\end{array}$ & [28-30] \\
\hline Isoliquiritigenin & & $\begin{array}{l}\text { (1) Cardioprotection against ischemic injury } \\
\text { (2) Decreased blood glucose level } \\
\text { (3) Prevention of neurodegenerative diseases } \\
\text { (4) Prevention of neuronal apoptotic death } \\
\text { (5) Repression of liver X receptor- } \alpha \text {-dependent } \\
\text { hepatic steatosis } \\
\text { (6) Induction of apoptosis } \\
\text { (7) Inhibition of cancer cell proliferation } \\
\text { (8) Enhancement of HepG2 cell radiosensitivity }\end{array}$ & {$[3,13,31-38]$} \\
\hline Naringin & & $\begin{array}{l}\text { (1) Inhibition of ROS-activated MAPK pathway } \\
\text { (2) Antihypertensive and antithrombotic } \\
\text { effects } \\
\text { (3) Protection of endothelial function from } \\
\text { ROS } \\
\text { (4) Protection against myocardial ischemia } \\
\text { reperfusion injury } \\
\text { (5) Antidiabetic effect } \\
\text { (6) Decreased inflammatory cell infiltration } \\
\text { (7) Neuroprotective effect } \\
\text { (8) Anti-inflammatory effect } \\
\text { (9) Protection against pulmonary fibrosis } \\
\text { (10) Induction of cancer cell apoptosis } \\
\text { (11) Attenuation of chronic pulmonary } \\
\text { neutrophilic inflammation } \\
\text { (12) Mitigation of erythrocyte aging }\end{array}$ & $\begin{array}{c}{[4-} \\
6,14,15,39- \\
47]\end{array}$ \\
\hline
\end{tabular}


TABLe 2: Continued.

\begin{tabular}{|c|c|c|c|}
\hline $\begin{array}{l}\text { Antioxidant } \\
\text { ingredient }\end{array}$ & Structure of compound & Pharmacological activity & References \\
\hline Glabridin & & $\begin{array}{l}\text { (1) Antioxidation } \\
\text { (2) Protection against obesity-related metabolic } \\
\text { disorders } \\
\text { (3) Neuroprotective effect } \\
\text { (4) Anti-inflammatory effect } \\
\text { (5) Anticancer effect }\end{array}$ & $\begin{array}{c}{[7,16,17,48,} \\
49]\end{array}$ \\
\hline Isorhamnetin & $\mathrm{OH}$ & $\begin{array}{l}\text { (1) Inhibition of ROS-induced apoptotic } \\
\text { pathway } \\
\text { (2) Anti-ROS-induced cardiomyopathy } \\
\text { (3) Protection of hepatocytes against oxidative } \\
\text { stress } \\
\text { (4) Antioxidant and antiproliferative effects } \\
\text { (5) Modulation of the peroxisome } \\
\text { proliferator-activated receptor pathway in } \\
\text { gastric cancer }\end{array}$ & {$[8,50-54]$} \\
\hline Calycosin & & $\begin{array}{l}\text { (1) Neuroprotection } \\
\text { (2) Inhibition of breast cancer growth }\end{array}$ & {$[55,56]$} \\
\hline Pinocembrin & $\mathrm{OH}$ & $\begin{array}{l}\text { (1) Neurovascular protection } \\
\text { (2) Protection of cerebral ischemia } \\
\text { (3) Increased viability and mitochondrial } \\
\text { membrane potential of cultured rat cerebral } \\
\text { microvascular endothelial cells } \\
\text { (4) Protection of rat brain against oxidation } \\
\text { and apoptosis } \\
\text { (5) Abrogation of neurotoxin effects } \\
\text { (6) Improvement of rat cognitive impairments } \\
\text { (7) Anticarcinogenic effects } \\
\text { (8) Triggering mitochondrial apoptosis in } \\
\text { cancer cells }\end{array}$ & {$[57-60]$} \\
\hline Nicotiflorin & & $\begin{array}{l}\text { (1) Improvement of brain infarct volume and } \\
\text { neurological deficits } \\
\text { (2) Protection against memory dysfunction, } \\
\text { energy metabolism failure, and oxidative stress } \\
\text { (3) Protection against cerebral ischemic } \\
\text { damage }\end{array}$ & [61-63] \\
\hline Liquiritigenin & & $\begin{array}{l}\text { (1) Improvement of behavioral performance } \\
\text { and attenuation of neuronal loss in the brain } \\
\text { (2) Protection of human lung cells (A549) } \\
\text { (3) Choleretic effect and induction of hepatic } \\
\text { transporters and phase-II enzymes } \\
\text { (4) Induction of apoptosis in the mitochondrial } \\
\text { pathway } \\
\text { (5) Anti-inflammatory effect } \\
\text { (6) Protection of osteoblast against cytotoxicity }\end{array}$ & {$[64-68]$} \\
\hline
\end{tabular}


TABLE 2: Continued.

\begin{tabular}{|c|c|c|c|}
\hline $\begin{array}{l}\text { Antioxidant } \\
\text { ingredient }\end{array}$ & Structure of compound & Pharmacological activity & References \\
\hline Kaempferol & & $\begin{array}{l}\text { (1) Attenuation of neuronal toxicity } \\
\text { (2) Inhibition of monoamine oxidase and } \\
\text { excessive NO production }\end{array}$ & [63] \\
\hline Licoflavone & & (1) Suppression of gastric mucosa injury & [69] \\
\hline Isobavachalcone & $\mathrm{OH}$ & $\begin{array}{l}\text { (1) Antioxidant effects on rat liver microsomes } \\
\text { and mitochondria } \\
\text { (2) Induction of neuroblastoma apoptosis }\end{array}$ & {$[70,71]$} \\
\hline Morusin & & (1) Anticancer properties & {$[72,73]$} \\
\hline Licochalcone A & & $\begin{array}{l}\text { (1) Antitumor and antimetastatic properties } \\
\text { (2) Apoptosis-promotion } \\
\text { (3) Inhibition of inflammatory cytokines and } \\
\text { ROS production }\end{array}$ & {$[74-76]$} \\
\hline Licochalcone B & & (1) Inhibition of cancer cell proliferation & [77] \\
\hline Rutin & & $\begin{array}{l}\text { (1) Inhibition of primary humoral immune } \\
\text { response }\end{array}$ & {$[78]$} \\
\hline
\end{tabular}


TABLE 2: Continued.

$\begin{aligned} & \text { Antioxidant } \\ & \text { ingredient }\end{aligned}$
Quercetin

of inflammatory cytokines and ROS [76]. Quercetin and kaempferol can modulate the degranulation and oxidative burst of stimulated human neutrophils. Rutin, which is derived from licorice, inhibits the primary humoral immune response in mice [78]. The induction of HO-1 by isorhamnetin may reduce ROS production, and the antioxidant property of isorhamnetin might inhibit the COX-2 expression in response to inflammation [54].

2.8. Others. The Sijunzi soup has a significant role in other aspects, such as the mitigation of musculoskeletal diseases and the progression of hematological system diseases.

The modulation of PI3K antioxidant effects and the attenuation of mitochondrial dysfunction by liquiritigenin represent an important mechanism for the protection of osteoblasts against cytotoxicity, which results from mitochondrial oxidative stress [68].

Naringin mitigates erythrocyte aging induced by paclitaxel (PTX), which suggests that naringin inhibits PTXinduced aging by reducing the PTX-induced oxidative stress [47].

\section{Antioxidants List}

See Tables 1 and 2 .

\section{Conclusion}

The oxidative products of organisms cause several diseases. Several studies have been conducted on the effects of antioxidant-scavenging free radical. Sijunzi decoction contains a variety of antioxidants. The effects of these antioxidants have gained attention from scholars at home and abroad. Various research results have been obtained. The antioxidants in Sijunzi decoction decrease free radical content and enhance the free radical scavenging activities in the body by adjusting the activity of the antioxidative enzyme system when spleen deficiency occurs. The antioxidants in Sijunzi decoction eliminate the peroxidation damage in tissues, cells, and various types of biological macromolecules and protect the normal structure and function of the cell membrane. Sijunzi decoction improves the pathological changes of tissues and cells in the cardiovascular and respiratory systems, corrects the nerve and endocrine functions, strengthens digestion, and induces tumor cell apoptosis. Revealing mainly the antioxidant of Sijunzi decoction is necessary to understand its antioxidant effects. Research to discover the antioxidant components of Sijunzi decoction and their target research are areas of future study in this field.

\section{Conflict of Interests}

The authors declare that there is no conflict of interests regarding the publication of this paper.

\section{References}

[1] Y. Wei and W. Li, "The c orrelation of experimental spleen deficiency and free radical metabolism and the adjustment of sijunzi decoction," Acta Veterinaria et Zootechnica Sinica, vol. 33, no. 1, pp. 100-104, 2002.

[2] W. Yanming and L. Wenguang, "Si Jun Zi decoction anti lipid peroxidation in vitro and the role of reactive oxygen free radicals," Acta Veterinaria et Zootechnica Sinica, vol. 33, no. 2, pp. 197-199, 2002.

[3] X. Zhang, P. Zhu, Y. Ma et al., "Natural antioxidant-isoliquiritigenin ameliorates contractile dysfunction of hypoxic cardiomyocytes via AMPK signaling pathway," Mediators of Inflammation, vol. 2013, Article ID 390890, 10 pages, 2013.

[4] J. Chen, R. Guo, H. Yan et al., "Naringin inhibits ROS-activated MAPK pathway in high glucose-induced injuries in $\mathrm{H} 9 \mathrm{c} 2$ cardiac cells," Basic \& Clinical Pharmacology \& Toxicology, vol. 114, no. 4, pp. 293-304, 2014

[5] M. Ikemura, Y. Sasaki, J. C. Giddings, and J. Yamamoto, "Preventive effects of hesperidin, glucosyl hesperidin and naringin on hypertension and cerebral thrombosis in stroke-prone spontaneously hypertensive Rats," Phytotherapy Research, vol. 26, no. 9, pp. 1272-1277, 2012.

[6] N. Rani, S. Bharti, M. Manchanda et al., "Regulation of heat shock proteins 27 and 70, p-Akt/p-eNOS and MAPKs by Naringin Dampens myocardial injury and dysfunction in vivo after ischemia/reperfusio," PLoS ONE, vol. 8, Article ID e82577, 2013.

[7] B. Fuhrman, S. Buch, J. Vaya et al., "Licorice extract and its major polyphenol glabridin protect low-density lipoprotein against lipid peroxidation: in vitro and ex vivo studies in humans and in atherosclerotic apolipoprotein E-deficient mice," The American Journal of Clinical Nutrition, vol. 66, no. 2, pp. 267-275, 1997.

[8] B. Sun, G. Sun, J. Xiao et al., "Isorhamnetin inhibits $\mathrm{H}_{2} \mathrm{O}_{2}$ induced activation of the intrinsic apoptotic pathway in $\mathrm{H} 9 \mathrm{c} 2$ cardiomyocytes through scavenging reactive oxygen species and ERK inactivation," Journal of Cellular Biochemistry, vol. 113, no. 2, pp. 473-485, 2012. 
[9] L. L. Pan and M. Dai, "Paeonol from Paeonia suffruticosa prevents TNF- $\alpha$-induced monocytic cell adhesion to rat aortic endothelial cells by suppression of VCAM-1 expression," Phytomedicine, vol. 16, no. 11, pp. 1027-1032, 2009.

[10] Y.-Q. Wang, M. Dai, J.-C. Zhong, and D.-K. Yin, "Paeonol inhibits oxidized low density lipoprotein-induced monocyte adhesion to vascular endothelial cells by inhibiting the mitogen activated protein kinase pathway," Biological and Pharmaceutical Bulletin, vol. 35, no. 5, pp. 767-772, 2012.

[11] H. Li, Y. H. Xie, Q. Yang et al., "Cardioprotective effect of paeonol and danshensu combination on isoproterenol-induced myocardial injury in rats," PloS ONE, vol. 7, no. 11, Article ID e48872, 2012.

[12] Q. Yang, S. Wang, Y. Xie et al., "Effect of Salvianolic acid b and paeonol on blood lipid metabolism and hemorrheology in myocardial schemia rabbits induced by pituitruin," International Journal of Molecular Sciences, vol. 11, no. 10, pp. 36963704, 2010.

[13] R. Gaur, K. S. Yadav, R. K. Verma, N. P. Yadav, and R. S. Bhakuni, "In vivo anti-diabetic activity of derivatives of isoliquiritigenin and liquiritigenin," Phytomedicine, vol. 21, no. 4, pp. 415-422, 2014.

[14] A. M. Mahmoud, M. B. Ashour, A. Abdel-Moneim, and O. M. Ahmed, "Hesperidin and naringin attenuate hyperglycemiamediated oxidative stress and proinflammatory cytokine production in high fat fed/streptozotocin-induced type 2 diabetic rats," Journal of Diabetes and its Complications, vol. 26, no. 6, pp. 483-490, 2012.

[15] M. A. Alam, K. Kauter, and L. Brown, "Naringin improves diet-induced cardiovascular dysfunction and obesity in high carbohydrate, high fat diet-fed rats," Nutrients, vol. 5, no. 3, pp. 637-650, 2013.

[16] P. Hasanein, "Glabridin as a major active isoflavan from Glycyrrhiza glabra (licorice) reverses learning and memory deficits in diabetic rats," Acta Physiologica Hungarica, vol. 98, no. 2, pp. 221-230, 2011.

[17] J. Lee, S. S. Choe, H. Jang et al., "AMPK activation with glabridin ameliorates adiposity and lipid dysregulation in obesity," Journal of Lipid Research, vol. 53, no. 7, pp. 1277-1286, 2012.

[18] J. Liu, L. Feng, D. Ma et al., "Neuroprotective effect of paeonol on cognition deficits of diabetic encephalopathy in streptozotocin-induced diabetic rat," Neuroscience Letters, vol. 549, pp. 63-68, 2013.

[19] N. N. Song, J. B. Wu, X. B. Wei, H. S. Guan, and X. M. Zhang, "Paeonol attenuates oxygen-glucose deprivation injury and inhibits NMDA receptor activation of cultured rat hippocampal neurons," Yao Xue Xue Bao, vol. 44, pp. 1228-1232, 2009.

[20] Y. W. Kim, H. E. Kang, M. G. Lee et al., "Liquiritigenin, a flavonoid aglycone from licorice, has a choleretic effect and the ability to induce hepatic transporters and phase-II enzymes," American Journal of Physiology: Gastrointestinal and Liver Physiology, vol. 296, no. 2, pp. G372-G381, 2009.

[21] N. Li, L. Fan, G. Sun et al., "Paeonol inhibits tumor growth in gastric cancer in vitro and in vivo," World Journal of Gastroenterology, vol. 16, no. 35, pp. 4483-4490, 2010.

[22] X. Yang, L. Zhang, S. Jiang, P. Gong, and F. Zeng, "Effect of dauricine on apoptosis and expression of apoptogenic protein after transient focal cerebral ischemia-reperfusion injury in rats," Zhongguo Zhong Yao Za Zhi, vol. 34, no. 1, pp. 78-83, 2009.

[23] H. Jin, S. Shen, X. Chen, D. Zhong, and J. Zheng, "CYP3Amediated apoptosis of dauricine in cultured human bronchial epithelial cells and in lungs of CD-1 mice," Toxicology and Applied Pharmacology, vol. 261, no. 3, pp. 248-254, 2012.

[24] C. Punvittayagul, R. Wongpoomchai, S. Taya, and W. Pompimon, "Effect of pinocembrin isolated from Boesenbergia pandurata on xenobiotic-metabolizing enzymes in rat liver," Drug Metabolism Letters, vol. 5, no. 1, pp. 1-5, 2011.

[25] J. Wang, Y. Li, X. Zu, M. Chen, and L. Qi, "Dauricine can inhibit the activity of proliferation of urinary tract tumor cells," Asian Pacific Journal of Tropical Medicine, vol. 5, no. 12, pp. 973-976, 2012.

[26] Z. Yang, C. Li, X. Wang et al., "Dauricine induces apoptosis, inhibits proliferation and invasion through inhibiting NF- $\kappa \mathrm{B}$ signaling pathway in colon cancer cells," Journal of Cellular Physiology, vol. 225, no. 1, pp. 266-275, 2010.

[27] X. D. Tang, X. Zhou, and K. Y. Zhou, "Dauricine inhibits insulin-like growth factor-I-induced hypoxia inducible factor $1 \alpha$ protein accumulation and vascular endothelial growth factor expression in human breast cancer cells," Acta Pharmacologica Sinica, vol. 30, no. 5, pp. 605-616, 2009.

[28] A. McLachlan, N. Kekre, J. McNulty, and S. Pandey, "Pancratistatin: A natural anti-cancer compound that targets mitochondria specifically in cancer cells to induce apoptosis," Apoptosis, vol. 10, no. 3, pp. 619-630, 2005.

[29] C. Griffin, A. Karnik, J. McNulty, and S. Pandey, "Pancratistatin selectively targets cancer cell mitochondria and reduces growth of human colon tumor xenografts," Molecular Cancer Therapeutics, vol. 10, no. 1, pp. 57-68, 2011.

[30] C. Griffin, J. McNulty, and S. Pandey, "Pancratistatin induces apoptosis and autophagy in metastatic prostate cancer cells," International Journal of Oncology, vol. 38, no. 6, pp. 1549-1556, 2011.

[31] E. J. Yang, J. S. Min, H. Y. Ku et al., "Isoliquiritigenin isolated from Glycyrrhiza uralensis protects neuronal cells against glutamate-induced mitochondrial dysfunction," Biochemical and Biophysical Research Communications, vol. 421, no. 4, pp. 658-664, 2012.

[32] H. K. Lee, E. Yang, J. Y. Kim, K. Song, and Y. H. Seong, "Inhibitory effects of glycyrrhizae radix and its active component, isoliquiritigenin, on $\mathrm{A} \beta(25-35)$-induced neurotoxicity in cultured rat cortical neurons," Archives of Pharmacal Research, vol. 35, no. 5, pp. 897-904, 2012.

[33] Y. M. Kim, T. H. Kim, Y. W. Kim et al., "Inhibition of liver X receptor- $\alpha$-dependent hepatic steatosis by isoliquiritigenin, a licorice antioxidant flavonoid, as mediated by JNK1 inhibition," Free Radical Biology \& Medicine, vol. 49, no. 11, pp. 1722-1734, 2010.

[34] X. Yuan, B. Yu, Y. Wang et al., "Involvement of endoplasmic reticulum stress in isoliquiritigenin-induced SKOV-3 cell apoptosis," Recent Patents on Anti-Cancer Drug Discovery, vol. 8, no. 2, pp. 191-199, 2013.

[35] X. Yuan, B. Zhang, N. Chen et al., "Isoliquiritigenin treatment induces apoptosis by increasing intracellular ROS levels in HeLa cells," Journal of Asian Natural Products Research, vol. 14, no. 8, pp. 789-798, 2012.

[36] X. Zhang, E. D. Yeung, J. Wang et al., "Isoliquiritigenin, a natural anti-oxidant, selectively inhibits the proliferation of prostate cancer cells," Clinical and Experimental Pharmacology and Physiology, vol. 37, no. 8, pp. 841-847, 2010.

[37] X. Chen, B. Zhang, X. Yuan et al., "Isoliquiritigenin-induced differentiation in mouse melanoma B16F0 cell line," Oxidative Medicine and Cellular Longevity, vol. 2012, Article ID 534934, 11 pages, 2012. 
[38] H. Chen, B. Zhang, X. Yuan et al., "Isoliquiritigenin-induced effects on Nrf2 mediated antioxidant defence in the HL-60 cell monocytic differentiation," Cell Biology International, vol. 37, no. 11, pp. 1215-1224, 2013.

[39] K. Gopinath and G. Sudhandiran, "Naringin modulates oxidative stress and inflammation in 3-nitropropionic acid-induced neurodegeneration through the activation of nuclear factorerythroid 2-related factor-2 signalling pathway," Neuroscience, vol. 227, pp. 134-143, 2012.

[40] K. Gopinath, D. Prakash, and G. Sudhandiran, "Neuroprotective effect of naringin, a dietary flavonoid against 3Nitropropionic acid-induced neuronal apoptosis," Neurochemistry International, vol. 59, no. 7, pp. 1066-1073, 2011.

[41] Y.-L. Luo, C.-C. Zhang, P.-B. Li et al., "Naringin attenuates enhanced cough, airway hyperresponsiveness and airway inflammation in a guinea pig model of chronic bronchitis induced by cigarette smoke," International Immunopharmacology, vol. 13, no. 3, pp. 301-307, 2012.

[42] Y. Chen, Y. Nie, Y. Luo et al., "Protective effects of naringin against paraquat-induced acute lung injury and pulmonary fibrosis in mice," Food and Chemical Toxicology, vol. 58, pp. 133140, 2013.

[43] E. Ramesh and A. A. Alshatwi, "Naringin induces death receptor and mitochondria-mediated apoptosis in human cervical cancer (SiHa) cells," Food and Chemical Toxicology, vol. 51, no. 1, pp. 97-105, 2013.

[44] H. Li, B. Yang, J. Huang et al., "Naringin inhibits growth potential of human triple-negative breast cancer cells by targeting $\beta$ catenin signaling pathway," Toxicology Letters, vol. 220, no. 3, pp. 219-228, 2013.

[45] P. L. Sequetto, T. T. Oliveira, I. R. Maldonado et al., "Naringin accelerates the regression of pre-neoplastic lesions and the colorectal structural reorganization in a murine model of chemical carcinogenesis," Food and Chemical Toxicology, vol. 64, pp. 200-209, 2014.

[46] Y. Nie, H. Wu, P. Li et al., "Anti-inflammatory effects of naringin in chronic pulmonary neutrophilic inflammation in cigarette smoke-exposed rats," Journal of Medicinal Food, vol. 15, no. 10, pp. 894-900, 2012.

[47] G. I. Harisa, "Naringin mitigates erythrocytes aging induced by Paclitaxel: an in vitro study," Journal of Biochemical and Molecular Toxicology, vol. 28, no. 3, pp. 129-136, 2014.

[48] X. Q. Yu, C. C. Xue, Z. W. Zhou et al., "In vitro and in vivo neuroprotective effect and mechanisms of glabridin, a major active isoflavan from Glycyrrhiza glabra (licorice)," Life Sciences, vol. 82, no. 1-2, pp. 68-78, 2008.

[49] Y. L. Hsu, L. Y. Wu, M. F. Hou et al., "Glabridin, an isoflavan from licorice root, inhibits migration, invasion and angiogenesis of MDA-MB-231 human breast adenocarcinoma cells by inhibiting focal adhesion kinase/Rho signaling pathway," Molecular Nutrition \& Food Research, vol. 55, no. 2, pp. 318-327, 2011.

[50] J. H. Yang, B. Y. Shin, J. Y. Han et al., "Isorhamnetin protects against oxidative stress by activating Nrf2 and inducing the expression of its target genes," Toxicology and Applied Pharmacology, vol. 274, pp. 293-301, 2014.

[51] C. Shi, L. Y. Fan, Z. Cai, Y. Y. Liu, and C. L. Yang, "Cellular stress response in Eca-109 cells inhibits apoptosis during early exposure to isorhamnetin.", Neoplasma, vol. 59, no. 4, pp. 361369, 2012.

[52] J. Boubaker, M. B. Sghaier, I. Skandrani, K. Ghedira, and L. Chekir-Ghedira, "Isorhamnetin 3-O-robinobioside from
Nitraria retusa leaves enhance antioxidant and antigenotoxic activity in human chronic myelogenous leukemia cell line K562," BMC Complementary and Alternative Medicine, vol. 12, article 135, 2012.

[53] L. Ramachandran, K. A. Manu, M. K. Shanmugam et al., "Sethi G.Isorhamnetin inhibits proliferation and invasion and induces apoptosis through the modulation of peroxisome proliferatoractivated receptor? activation pathway in gastric cancer," The Journal of Biological Chemistry, vol. 287, pp. 38028-38040, 2012.

[54] K. Seo, J. H. Yang, S. C. Kim, S. K. Ku, S. H. Ki, and S.M. Shin, "The antioxidant effects of isorhamnetin contribute to inhibit COX-2 expression in response to inflammation: a potential role of HO-1," Inflammation, vol. 37, no. 3, pp. 712-722, 2014.

[55] C. Guo, L. Tong, M. Xi, H. Yang, H. Dong, and A. Wen, "Neuroprotective effect of calycosin on cerebral ischemia and reperfusion injury in rats," Journal of Ethnopharmacology, vol. 144, no. 3, pp. 768-774, 2012.

[56] J. Chen, X. Zhao, Y. Ye, Y. Wang, and J. Tian, "Estrogen receptor beta-mediated proliferative inhibition and apoptosis in human breast cancer by calycosin and formononetin," Cellular Physiology and Biochemistry, vol. 32, no. 6, pp. 1790-1797, 2013.

[57] M. Gao, R. Liu, S. Y. Zhu, and G. H. Du, "Acute neurovascular unit protective action of pinocembrin against permanent cerebral ischemia in rats," Journal of Asian Natural Products Research, vol. 10, no. 6, pp. 551-558, 2008.

[58] J. Qian, "Cardiovascular pharmacological effects of bisbenzylisoquinoline alkaloid derivatives," Acta Pharmacologica Sinica, vol. 23, no. 12, pp. 1086-1092, 2002.

[59] D. Kong, F. Zhang, D. Wei et al., "Paeonol inhibits hepatic fibrogenesis via disrupting nuclear factor- $\kappa \mathrm{B}$ pathway in activated stellate cells: in vivo and in vitro studies," Journal of Gastroenterology and Hepatology, vol. 28, no. 7, pp. 1223-1233, 2013.

[60] M. A. S. Kumar, M. Nair, P. S. Hema, J. Mohan, and T. R. Santhoshkumar, "Pinocembrin triggers Bax-dependent mitochondrial apoptosis in colon cancer cells," Molecular Carcinogenesis, vol. 46, no. 3, pp. 231-241, 2007.

[61] R. Li, M. Guo, G. Zhang, X. Xu, and Q. Li, "Nicotiflorin reduces cerebral ischemic damage and upregulates endothelial nitric oxide synthase in primarily cultured rat cerebral blood vessel endothelial cells," Journal of Ethnopharmacology, vol. 107, no. 1, pp. 143-150, 2006.

[62] A. Prakash, B. Shur, and A. Kumar, "Naringin protects memory impairment and mitochondrial oxidative damage against aluminum-induced neurotoxicity in rats," International Journal of Neuroscience, vol. 123, no. 9, pp. 636-645, 2013.

[63] R. Li, M. Guo, G. Zhang, X. Xu, and Q. Li, "Neuroprotection of nicotiflorin in permanent focal cerebral ischemia and in neuronal cultures," Biological and Pharmaceutical Bulletin, vol. 29, no. 9, pp. 1868-1872, 2006.

[64] R. T. Liu, L. B. Zou, J. Y. Fu, and Q. J. Lu, “Effects of liquiritigenin treatment on the learning and memory deficits induced by amyloid $\beta$-peptide (25-35) in rats," Behavioural Brain Research, vol. 210, no. 1, pp. 24-31, 2010.

[65] X. H. Dai, H. E. Li, C. J. Lu et al., "Liquiritigenin prevents Staphylococcus aureus-mediated lung cell injury via inhibiting the production of $\alpha$-hemolysin," Journal of Asian Natural Products Research, vol. 15, no. 4, pp. 390-399, 2013.

[66] M. Zhou, H. Higo, and Y. Cai, "Inhibition of hepatoma 22 tumor by liquiritigenin," Phytotherapy Research, vol. 24, no. 6, pp. 827833, 2010. 
[67] Y. W. Kim, R. J. Zhao, S. J. Park et al., "Anti-inflammatory effects of liquiritigenin as a consequence of the inhibition of NF- $\kappa \mathrm{B}-$ dependent iNOS and proinflammatory cytokines production," British Journal of Pharmacology, vol. 154, no. 1, pp. 165-173, 2008.

[68] E. M. Choi, K. S. Suh, and Y. S. Lee, "Liquiritigenin restores osteoblast damage through regulating oxidative stress and mitochondrial dysfunction," Phytotherapy Research, vol. 28, no. 6, pp. 880-886, 2014.

[69] X. C. Lin, Y. Y. Chen, S. T. Bai, J. Zheng, and L. Tong, "Protective effect of licoflavone on gastric mucosa in rats with chronic superficial gastritis," Nan Fang Yi Ke Da Xue Xue Bao, vol. 33, pp. 299-304, 2013.

[70] H. Haraguchi, J. Inoue, Y. Tamura, and K. Mizutani, "Antioxidative components of Psoralea corylifolia (Leguminosae)," Phytotherapy Research, vol. 16, no. 6, pp. 539-544, 2002.

[71] R. Nishimura, K. Tabata, M. Arakawa et al., "Isobavachalcone, a chalcone constituent of Angelica keiskei, induces apoptosis in neuroblastoma," Biological and Pharmaceutical Bulletin, vol. 30, no. 10, pp. 1878-1883, 2007.

[72] J. Lee, S. Won, C. Chao et al., "Morusin induces apoptosis and suppresses NF- $\kappa$ B activity in human colorectal cancer HT-29 cells," Biochemical and Biophysical Research Communications, vol. 372, no. 1, pp. 236-242, 2008.

[73] L. Wang, H. Guo, L. Yang et al., "Morusin inhibits human cervical cancer stem cell growth and migration through attenuation of NF- $\kappa$ B activity and apoptosis induction," Molecular and Cellular Biochemistry, vol. 379, no. 1-2, pp. 7-18, 2013.

[74] M. M. Rafi, R. T. Rosen, A. Vassil et al., "Modulation of bcl-2 and cytotoxicity by licochalcone-A, a novel estrogenic flavonoid," Anticancer Research, vol. 20, no. 4, pp. 2653-2658, 2000.

[75] X. Yuan, D. Li, H. Zhao et al., "Licochalcone A-induced human bladder cancer T24 cells apoptosis triggered by mitochondria dysfunction and endoplasmic reticulum stress," BioMed Research International, vol. 2013, Article ID 474272, 9 pages, 2013.

[76] J. Kim, E. K. Shin, J. H. Park, Y. H. Kim, and J. H. Y. Park, "Antitumor and antimetastatic effects of licochalcone A in mouse models," Journal of Molecular Medicine, vol. 88, no. 8, pp. 829-838, 2010.

[77] X. Yuan, T. Li, E. Xiao et al., "Licochalcone B inhibits growth of bladder cancer cells by arresting cell cycle progression and inducing apoptosis," Food and Chemical Toxicology, vol. 65, pp. 242-251, 2014.

[78] P. Akbay, A. A. Basaran, U. Undeger, and N. Basaran, "In vitro immunomodulatory activity of flavonoid glycosides from Urtica dioica L.," Phytotherapy Research, vol. 17, no. 1, pp. 34-37, 2003.

[79] P. M. Amália, M. N. Possa, M. C. Augusto, and L. S. Francisca, "Quercetin prevents oxidative stress in cirrhotic rats," Digestive Diseases and Sciences, vol. 52, no. 10, pp. 2616-2621, 2007.

[80] R. Liu, M. Gao, Z. Yang, and G. Du, "Pinocembrin protects rat brain against oxidation and apoptosis induced by ischemiareperfusion both in vivo and in vitro," Brain Research, vol. 1216, pp. 104-115, 2008.

[81] H.-M. Guang and G.-H. Du, "Protections of pinocembrin on brain mitochondria contribute to cognitive improvement in chronic cerebral hypoperfused rats," European Journal of Pharmacology, vol. 542, no. 1-3, pp. 77-83, 2006.

[82] Y. Wang, J. Gao, Y. Miao et al., "Pinocembrin protects SHSY5Y cells against MPP+-induced neurotoxicity through the mitochondrial apoptotic pathway," Journal of Molecular Neuroscience, 2014. 

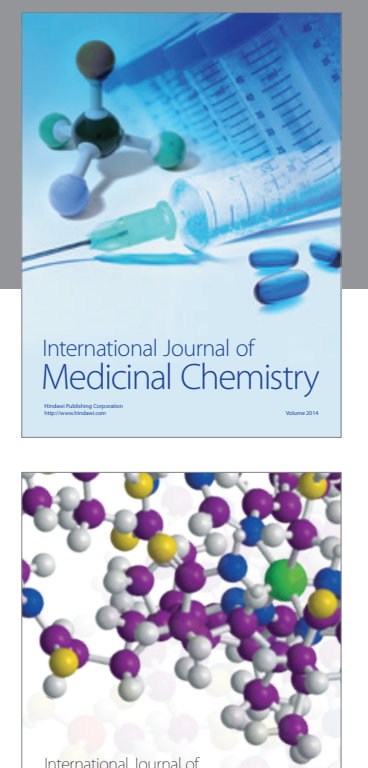

\section{Carbohydrate} Chemistry

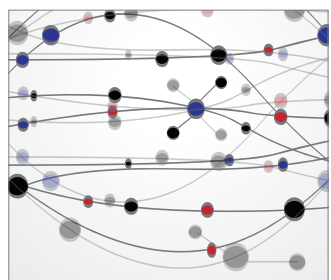

The Scientific World Journal
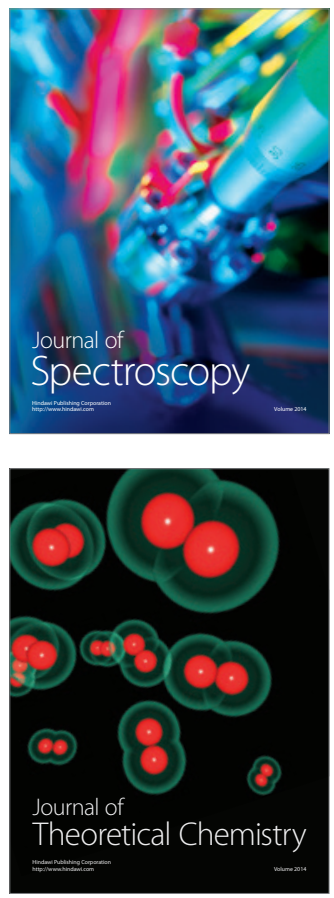
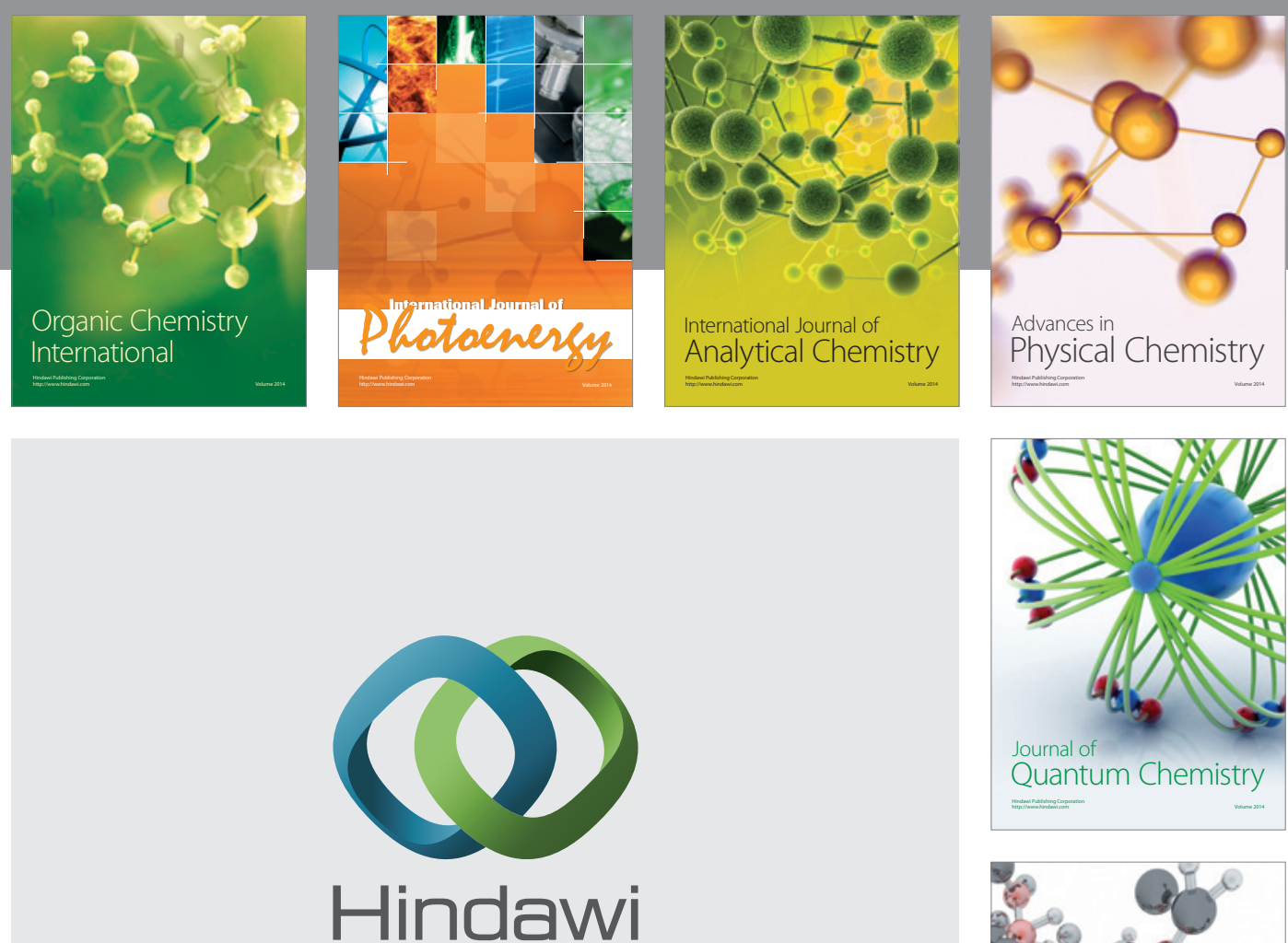

Submit your manuscripts at

http://www.hindawi.com

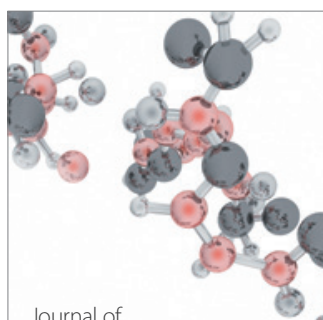

Analytical Methods

in Chemistry

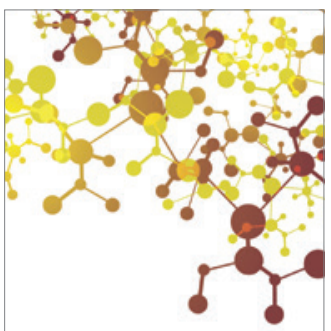

Journal of

Applied Chemistry

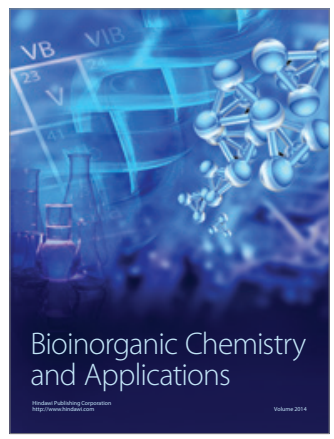

Inorganic Chemistry
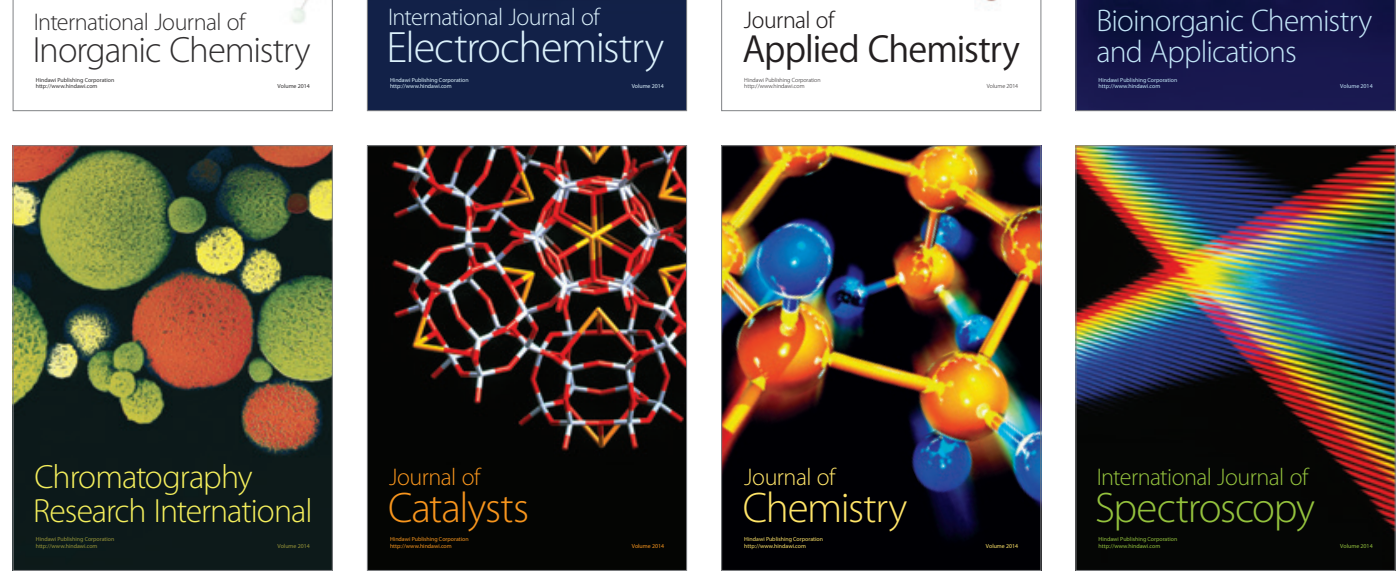